\title{
Fibrosis retroperitoneal secundaria a neoplasia gástrica
}

\author{
Oyarzabal Pérez I, Zubiaurre Lizarralde L*, Bujanda Fernández de Pierola L*, \\ Agirreazaldegi García L, Crespo Crespo I.
}

Servicio de Urología del Hospital Donostia. *Servicio de Digestivo del Hospital Donostia. San Sebastián.

Actas Urol Esp. 2008;32(3):345-347

\section{RESUMEN \\ FIBROSIS RETROPERITONEAL SECUNDARIA A NEOPLASIA GÁSTRICA}

La fibrosis retroperitoneal es una entidad muy poco frecuente que habitualmente engloba la aorta abdominal y los uréteres. Dos tercios de las veces es de causa idiopática y en un tercio es secundaria a otros procesos como fármacos, infecciones o enfermedades sistémicas y tumores de los que se estima puede ser responsables del 8 al 10\%. El más frecuente es el adenocarcinoma colorrectal, resultando anecdótica la localización gástrica con seis casos descritos hasta la fecha. Presentamos un caso de una paciente con cáncer gástrico y fibrosis retroperitoneal secundaria de difícil diagnóstico y manejo.

Palabras clave: Fibrosis retroperitoneal. Síndrome de Ormond. Neoplasia gástrica. Cáncer, Estómago. Ureterohidronefrosis bilateral.

\section{ABSTRACT}

FIBROSIS RETROPERITONEAL SECONDARY TO GASTRIC NEOPLASIA

The retroperitoneal fibrosis is a very little frequent pathology that habitually includes the abdominal aorta and ureters. Two thirds of cases the etiology is idiopathic and in a third it is secondary to other causes as drugs, generalized infections or diseases and tumors which are considered to be responsible from 8 to $10 \%$. The most frequent is colorectal adenocarcinoma, being anecdotal the gastric location with six described cases to date. We present a case of a patient with gastric cancer and secondary retroperitoneal fibrosis of difficult diagnosis and handling.

Keywords: Retroperitoneal fibrosis. Ormond’s sindrome. Gastric neoplasia. Cancer, Stomach, Bilateral ureterohidronefrosis.

$\mathrm{J}^{\mathrm{s}}$ ohn Kelso Ormod (1886-1979) urólogo nacido en Princeton, New Jersey, en 1948 describió 2 casos de una fibrosis retroperitoneal peculiar que englobaba a los dos uréteres ${ }^{1}$. Esto dio nombre a una entidad de patogenia desconocida, que se caracteriza por dolor lumbar progresivo, sintomatología abdominal e insuficiencia renal obstructiva ocasional por atrapamiento ureteral bilateral con medialización de los uréteres así como de otras estructuras retroperitoneales.

\section{CASO CLÍNICO}

Mujer de 55 años que acude por dolor abdominal de 3 meses de evolución. Tenía antecedente de tuberculosis (TBC) a los 8 años y estaba intervenida de apendicitis perforada a los 9 años. No recibía tratamiento ni tenía hábitos tóxicos. $\mathrm{El}$ dolor que las últimas dos semanas había disminuido en intensidad se había generalizado a todo el abdomen y posteriormente a la espalda. Mejoraba parcialmente con ibuprofeno, la comida y con omeprazol. Además la paciente presentaba, astenia, pérdida de $3 \mathrm{k}$ de peso en un mes y aumento del perímetro abdominal sin alteración del ritmo intestinal ni otra clínica digestiva, urinaria, respiratoria o ginecológica.

A la exploración destacaba una masa en fosa iliaca derecha en zona de cicatriz de apendicectomía pararrectal derecha. El abdomen era blando y no presentaba signos de irritación peritoneal.

Se realizó una analítica con bioquímica (creatinina, glucosa, triglicéridos, colesterol, amilasa, fosfatasa alcalina, AST, ALT, GGT, bilirrubina, proteinograma) hemograma, coagulación y estudio de hormonas tiroideas con un resultado normal. En la 
ecografía se objetivó una dilatación moderada en la pelvis renal izquierda y ascitis de escasa cuantía. Resto normal.

Ingresó en el hospital y a los 3 días destacaba una creatinina de 5,9; urea de 99, leucocitosis de 9.690 con $81 \%$ de neutrófilos y VSG de 40. GOT, GGT, FA, CPK y LDH seguían siendo normales. Se realizó una ecografía abdominal urgente donde se objetivó una hidronefrosis bilateral, más intensa en el lado izquierdo, ascitis y derrame pleural bilateral. Valorado por el servicio de urología solicitó una tomografía computerizada (TC) abdomino-pélvica y realizaron un cateterismo ureteral bilateral. La TC no aportó ningún hallazgo nuevo respecto a la ecografía salvo una hernia de Spiegel en pared abdominal derecha no complicada (Fig. 1).

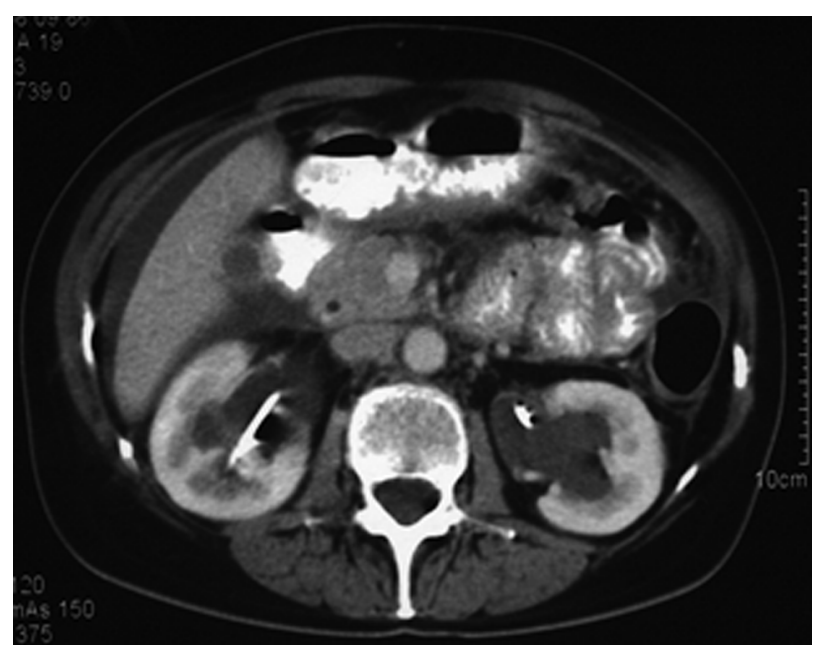

FIGURA 1. Puede apreciarse hidronefrosis bilateral, ascitis, engrosamiento de la pared gástrica y del colon transverso

La función renal evolucionó favorablemente normalizándose al décimo día, pero continuó con molestias abdominales y náuseas por lo que se solicitó estudio complementario mediante gastroscopia, ecografía ginecológica, colonoscopia, analítica con marcadores tumorales, radiografía $(\mathrm{RX})$ de tórax, BK en orina y paracentesis diagnóstica. De todas estas exploraciones destacaba un $\mathrm{Ca} 125$ de 441 (normal <35), un derrame pleural bilateral, un leve engrosamiento de pliegues gástricos con biopsias normales. El estudio del líquido ascítico resultó sero-hemático con 2.280 células (22\% PMN y 78\% de linfocitos), glucosa 104; 4,07 proteínas. Negativo para células malignas, cultivo y micobacterias. ADA 16,9 y Ca 125 de 598. En la colonoscopia había lesiones lesiones papulosas de 3 y $2 \mathrm{~cm}$ en colon transverso con biopsias en las que se observaba infiltrado inflamatorio.

Al mes del ingreso se planteó laparoscopia exploradora, que objetivó una pequeña herniación pararrectal derecha, escaso líquido peritoneal libre de aspecto turbio, signos inflamatorios del peritoneo y lesiones blanquecinas sobre peritoneo pélvico y diafragmático como cabezas de alfiler. Se tomaron biopsias de ovario y peritoneo. El estudio AP de los ovarios fue normal, de las biopsias del peritoneo tan sólo detectaron un mínimo componente inflamatorio sin células malignas y el nódulo blanquecino resultó ser tejido adiposo. El cultivo para micobacterias resultó negativo con un $\mathrm{ADA}<20$.

Se repitió la colonoscopia al mes y medio detectando una lesión papulosa de $3 \mathrm{~cm}$ que se biopsió con resultado AP normal y cultivo BK negativo. Ante la alta sospecha de TBC se inició tratamiento tuberculostático, sin embargo tras la ausencia de respuesta se suspendió a las 4 semanas. Posteriormente se inició tratamiento con prednisona $30 \mathrm{mg}$ al día por afectación ureteral bilateral sin evidencia de compresión tumoral y por lo tanto sospecha de fibrosis retroperitoneal idiopática, aunque no se había objetivado imagen clara de fibrosis en TC.

Finalmente, tras repasar la TC abdominal se visualizó un engrosamiento de la pared gástrica por lo que se realizó nueva gastroscopia con macrobiopsias a los 3 meses del ingreso, siendo el estudio AP de carcinoma con células en anillo de sello.

El diagnóstico final fue de cáncer gástrico con células en anillo de sello con infiltración de órganos vecinos, pared del colon transverso (T4NxMx) con obstrucción ureteral secundaria a fibrosis retroperitoneal paraneoplásica. Tras valorar el caso en sesión conjunta con oncología y cirugía general, se desestimó la posibilidad de cirugía radical y se decidió tratamiento quimioterápico paliativo, falleciendo la misma a los 21 meses del diagnóstico.

\section{DISCUSIÓN}

El cáncer gástrico es el quinto tumor más frecuente en España y es muy infrecuente su debut con crecimiento exofítico con infiltración del colon, es decir, sin detectar imagen de neoplasia en gastroscopia. Además es excepcional como causa de fibrosis retroperitoneal secundaria con menos de ocho casos publicados en la literatura. 
La fibrosis retroperitoneal o enfermedad de Ormond, se trata de una entidad que cursa con una reacción inflamatoria retroperitoneal que evoluciona a una fibrosis que habitualmente engloba la aorta abdominal y los uréteres. En el $67 \%$ de los casos es de causa idiopática. Generalmente es más frecuente en mujeres en la $5^{\mathrm{a}}-6^{\mathrm{a}}$ década de la vida, presentándose con ureterohidronefrosis bilateral y cuyo tratamiento se realiza con corticoides y derivación urinaria en los casos necesarios ${ }^{2}$. Por el contrario en un tercio de las veces esta enfermedad es secundaria a otros procesos como fármacos, infecciones o enfermedades sistémicas y tumores de los que se estima ser responsables del 8 al 10\% de los casos. El más frecuente es el adenocarcinoma colorrec$\mathrm{tal}^{3,4}$, siendo extrañas las citaciones en la bibliografía de localizaciones supraumbilicales ${ }^{5}$.

En un inicio se pensó en la posibilidad de TBC peritoneal como causa del cuadro basado en sus antecedentes, a pesar de tener un valor de ADA en líquido peritoneal menor de $20 \mathrm{U} / \mathrm{L}$ y no presentar otros factores predisponentes como ser VIH positivo, alcohólico, padecer otras neoplasias, estar gastrectomizado o ser paciente de edad avanzada. Entre el 4 y el 11\% de las TBC son de localización extrapulmonar y entre el 17 y el 25\% coexiste con afectación pulmonar. La TBC peritoneal se presenta en el 0,1$0,7 \%$ de todas.

No hay criterios unificados para su diagnóstico pero se cree que la TC o la RMN son las técnicas de elección donde habitualmente se observa una masa abdominal que engloba los uréteres y los grandes vasos cavo-aorto-ilíacos ${ }^{2}$. En este caso pasa desapercibido probablemente debido a que la paciente se presenta en una fase incipiente de la enfermedad, ya que al ingreso tan sólo tenía dilatación moderada del sistema pielocalicial izquierdo con función renal normal. Los reactantes de fase aguda (VSG y PCR) pueden ayudar pero es la evaluación histológica de dicha masa la que da el diagnóstico definitivo. El marcador Ca 125 no resulta útil en el diagnóstico etiológico. Se relaciona mayoritariamente con cáncer de ovario y se emplea en su diagnóstico y seguimiento pero hay otras muchas causas que elevan su valor: hepatopatía (en casi el 100\% de los casos puede estar elevado), hepatocarcinoma, TBC peritoneal, ascitis, lupus con síndrome nefrótico, embarazo, endometriosis, sarcoidosis y en múltiples tumores (melanoma, linfoma, endometrial, mama, cervix, colon, páncreas, pulmón, estómago, vías biliares, riñón, uretra, esófago y GIST).

Actualmente se desconoce si la fibrosis retroperitoneal en el contexto de una neoplasia es debida a un fenómeno inflamatorio reactivo o si por el contrario puede tratarse de una diseminación locorregional del propio tumor, pero sí parece relacionarse con un estadío evolutivo mayor de la enfermedad y por lo tanto le confiere una menor resecabilidad y peor pronóstico.

\section{REFERENCIAS}

1. Ormond J. Journal of Urology. 1948;59:1072

2. Vaglio A, Salvarani C and Buzio C. Retroperitoneal fibrosis. Lancet. 2006;367(9506):241-251.

3. Marinoni M, Boccasanta P, Strinna M, Venturi M. Diagnosis and treatment of retroperitoneal fibrosis and tumours. British Journal of Surgery. 1997;Suppl.84(2):109

4. Wu J, Catalano E, Coppola D. Retroperitoneal fibrosis (Ormond's disease): clinical pathologic study of eight cases. Cancer control. 2002;9(5):432-437.

5. Landaluce Olavarria A, Estraviz Mateos B, Gamarra Quintanilla M, Goicoechea Artola JM, Sarabia García S. Neoplasia gástrica y fibrosis retroperitoneal: ¿factor de mal pronóstico?. Cirugía Española. 2007;81(2):109-110.

Correspondencia autor: Dr. I. Oyarzabal Pérez Servicio de Urología. Hospital de Donostia Paseo Doctor Beguiristain, s/n - 20014 Donostia Tel: 943007021

E-mail autor: oyarzigor@yahoo.com Información artículo: Nota clínica

Trabajo recibido: abril 2007

Trabajo aceptado: mayo 2007 